\title{
TWISTED FORMS OF FINITE ÉTALE EXTENSIONS AND SEPARABLE POLYNOMIALS
}

\author{
FRANK DEMEYER
}

(Received 25 April 2000)

\begin{abstract}
Examples of twisted forms of finite étale extensions and separable polynomials are calculated using Mayer-Vietoris sequences for non-abelian cohomology.
\end{abstract}

2000 Mathematics Subject Classification. 13B05, 13B25, 13B40.

1. Introduction. Let $S$ be a finite étale extension of a commutative Noetherian ring $R$ (a finitely generated projective separable extension of $R$ ). A twisted form of $S$ (in the Zariski topology) is a finite étale extension $T$ of $R$ with $R_{P} \otimes S \cong R_{P} \otimes T$ as $R_{P}$-algebras for each prime ideal $P$ of $R$. In this case $S$ is locally isomorphic to T. If $Q \subset P$ are prime ideals of $R$ and $R_{P} \otimes S \cong R_{P} \otimes T$, then $R_{Q} \otimes S \cong R_{Q} \otimes T$ so prime can be replaced by maximal in the definition of the twisted form. In this paper, we study the set of isomorphism classes of twisted forms of $S$. We especially concentrate on the case where $S \cong R[t] /(p(t))$, where $p(t)$ is a separable polynomial in $R[t]$. Throughout this paper, $R$ denotes a commutative Noetherian ring.

We first observe the well-known facts that if $R$ is an integrally closed domain, then there are no twisted forms of $S$ and in general the twisted forms of $S$ are in bijective correspondence with $H^{1}(X$, Aut $(\mathscr{Y}))$, where $\operatorname{Aut}_{R}(\mathscr{Y})$ is the sheaf of automorphisms on $X=\operatorname{Spec}(R)$ associated to $\operatorname{Aut}_{R}(S)$. We check that $H^{1}(X$,Aut $(\mathscr{S}))$ is unchanged modulo a nilpotent ideal.

With some hypotheses on the sheaf $\operatorname{Aut}_{R}(\mathscr{S})$, when $R$ is a one-dimensional domain or if $R$ is a reduced one-dimensional ring with connected spectrum, $H^{1}(X$, Aut $(\mathscr{Y}))$ fits into a Mayer-Vietoris sequence which makes computations possible. These computations are the point of this article. For infinitely many prime numbers $p, q$ we give a class of examples of integral domains $R$ and separable polynomials $t^{p}-q \in R[t]$ with the cardinality of the set of isomorphism classes of twisted forms of $S \cong R[t] /(p(t))$ equal to $(p-1)$ !. When $p=3$ these twisted forms $T$ are isomorphic to algebras $T \cong \oplus_{j=0}^{2} I^{j} t^{j}$ with $t^{3}=q$ and $I$ is a fractional ideal with $I^{3}=R$. We give an example of twisted forms that do not have this structure. We also give one-dimensional rings over which finite étale extensions may not have either a primitive element nor a normal basis but which are twisted forms of extensions which do. We also give a separable polynomial which is irreducible over $R$ but factors into linear factors at each localization $R_{P}$ of $R$ and modulo each maximal ideal of $R$.

2. Mayer-Vietoris sequences and examples. Let $R$ be a commutative Noetherian ring and $S$ a finite étale $R$-algebra. Let $X=\operatorname{Spec}(R)$ be the space of prime ideals of $R$ 
with the Zariski topology and $\mathcal{O}_{X}$ the associated sheaf of rings on $X, \mathscr{Y}$ the sheaf of $O_{X^{-}}$ algebras associated to $S$ (see [5, pages 70 and 130]). For each open set $U \subset X$ associate to $U$ the group of $R$-algebra automorphisms $\operatorname{Aut}_{\mathscr{O}(U)}(\mathscr{S}(U))$, and if $V \subset U$ associate the natural restriction $\operatorname{Aut}_{\mathscr{O}(U)}(\mathscr{Y}(U)) \rightarrow \operatorname{Aut}_{\mathscr{O}(V)}(\mathscr{Y}(V))$. We begin by recording for the readers convenience that $\operatorname{Aut}_{R}(\mathscr{S})$ forms a sheaf on $X$ and give some of its properties (see also [3]).

LEMMA 2.1. Let $S$ be a finite étale $R$-algebra.

(a) If $U$ is an open set in $X$ and $\left\{V_{i}\right\}$ is an open cover of $U$, and if $\sigma \in \operatorname{Aut}_{\mathscr{O}(U)}(\mathscr{S}(U))$ satisfies $1 \otimes \sigma=1$ in $\operatorname{Aut}_{\mathscr{O}\left(V_{i}\right)}\left(\mathscr{Y}\left(V_{i}\right)\right)$ for all $i$, then $\sigma=1$ in $\operatorname{Aut}_{\mathscr{O}(U)}(\mathscr{Y}(U))$.

(b) If $U$ is an open set in $X$ and $\left\{V_{i}\right\}$ is an open cover of $U$, and if $\sigma_{i} \in \operatorname{Aut}_{\mathscr{O}\left(V_{i}\right)}\left(\mathscr{Y}\left(V_{i}\right)\right)$ with restrictions $\sigma_{i}=\sigma_{j}$ in $\operatorname{Aut}_{\mathscr{O}\left(V_{i} \cap V_{j}\right)}\left(\mathscr{Y}\left(V_{i} \cap V_{j}\right)\right)$, then there is an element $\sigma \in$ $\operatorname{Aut}_{\mathscr{O}(U)}(\mathscr{Y}(U))$ whose restriction to $\mathrm{Aut}_{\mathcal{O}\left(V_{i}\right)}\left(\mathscr{Y}\left(V_{i}\right)\right)$ is $\sigma_{i}$.

LEMMA 2.2. Let $S, T$ be finite étale $R$-algebras and $P$ a prime ideal in $R$. Let $\sigma$ : $R_{P} \otimes S \rightarrow R_{P} \otimes T$ be an $R_{P}$-algebra homomorphism. Then there is an open set $U$ in $X$

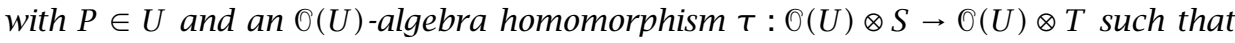
$1 \otimes \tau=\sigma \in \operatorname{Alg}_{R_{P}}\left(R_{P} \otimes S, R_{P} \otimes T\right)$. If $\sigma$ is an isomorphism then $U$ can be chosen so $\tau$ is an isomorphism.

If $S, T$ are finite étale $R$-algebras and $R_{P} \otimes S \cong R_{P} \otimes T$ for all prime ideals $P$ of $R$ we say that $S$ and $T$ are locally isomorphic or that $T$ is a twisted form of $S$. If $T$ is a twisted form of $S$ then Lemma 2.2 implies there is an open cover $U=\left\{U_{i}\right\}$ of $X$ and isomorphisms $\sigma_{i}: \mathcal{O}\left(U_{i}\right) \otimes S \rightarrow \mathcal{O}\left(U_{i}\right) \otimes T$ for all $i$. Define an element $a \in Z^{1}(\mathcal{U}$, Aut $(\mathscr{S}))$ by assigning to the index pair $i, j$ the automorphism $a(i, j)=\sigma_{i}^{-1} \sigma_{j} \in \operatorname{Aut}_{\mathscr{O}\left(U_{i} \cap U_{j}\right)}\left(\mathcal{O}_{(}\left(U_{i} \cap\right.\right.$ $\left.U_{j}\right) \otimes S$ ). Passing to the limit over all covers of $X$ gives an injection from the set of isomorphism classes of twisted forms of $S$ to $H^{1}(X$, Aut $(\mathscr{S})$ ). By descent, (see [7, 2.2, page 110] or [8, page 19]), this assignment is onto so $H^{1}(X, \operatorname{Aut}(\mathscr{Y}))$ classifies the twisted forms of $S$. In the next result we point out that, as with the Brauer group, there are no nontrivial twisted forms in the geometrically irreducible case.

Proposition 2.3. If $R$ is an integrally closed domain and $S$ is a finite étale $R$-algebra, then $H^{1}(X, \operatorname{Aut}(\mathscr{S}))=\{1\}$.

Proof. We can write $S=S_{1} \oplus \cdots \oplus S_{k}$, where each $S_{i}$ has a connected spectrum. By [6, Theorem 4.3] or [8, Proposition 3.19, page 28], each $S_{i}$ is an integrally closed domain. Let $K$ be the quotient field of $R$. Then $K \otimes S=\oplus_{i=1}^{k} K \otimes S_{i}$, where each $K \otimes S_{i}$ is a finite-dimensional separable field extension of $K$, and $S_{i}$ is the integral closure of $R$ in $K \otimes S_{i}$. Let $\sigma \in \operatorname{Aut}_{K}(K \otimes S)$, then since the image of an integral element is integral, $\left.\sigma\right|_{S} \in \operatorname{Aut}_{R}(S)$ and $\sigma=\left.1 \otimes \sigma\right|_{S}$. Therefore, the natural map $\operatorname{Aut}_{R}(S) \rightarrow \operatorname{Aut}_{K}(K \otimes S)$ is a bijection which implies that the sheaf $\operatorname{Aut}(\mathscr{Y})$ is constant. Therefore, $H^{1}(X$, $\operatorname{Aut}(\mathscr{Y}))=$ $\{1\}$.

COROLLARY 2.4. Let $R$ be an integrally closed domain and $S, T$ finite étale $R$-algebras. If $R_{p} \otimes S \cong R_{P} \otimes T$ as $R_{P}$-algebras for each $P \in \operatorname{Spec}(R)$, then $S \cong T$ as $R$-algebras.

LEMMA 2.5. Let $S$ be a finite étale $R$-algebra, $I$ an ideal in $R$, and $\rho: \operatorname{Aut}_{R}(S) \rightarrow$ $\operatorname{Aut}_{R / I}(S / I)$ the natural map. 
(a) If $R$ has a connected spectrum then $\rho$ is a one-to-one map.

(b) If I is nilpotent then $\rho$ is a bijection map.

Proof. (a) Assume first that $S$ is connected and Galois over $R$. Then $\operatorname{Aut}_{R}(S)=$ Galois group of $S$ over $R=$ the Galois group of $S / I S$ over $R / I \subset \operatorname{Aut}_{R / I}(S / I S)$ so $\rho$ is a one-to-one map in this case. If $S$ is connected but not necessarily Galois, imbed $S$ in a connected Galois extension $N$ of $R$ (see [2, Theorem 3.2.9]). Every $R$-automorphism of $S$ extends to an automorphism of $N$, and any two such extensions differ by an element of $H=\left\{\sigma \in \operatorname{Aut}_{R}(N)|\sigma|_{S}=1\right\}$ (see [2, Chapter 3]). By flatness, $S / I S$ is a subalgebra of $N / I N$ and $H$ is the subgroup of the Galois group of $N / I N$ over $R / I$ fixing $S / I S$. If $\tau, \sigma \in \operatorname{Aut}_{R}(S)$ with extensions $\bar{\tau}, \bar{\sigma}$ to $N$ and with the natural image of $\tau=\sigma$ in $\operatorname{Aut}_{R / I}(S / I S)$, then $\bar{\tau}^{-1} \bar{\sigma} \in H$ so $\tau=\sigma$ on $S$ and $\rho$ is a one-to-one map in this case.

If $R$ is connected then $S=S e_{1} \oplus \cdots \oplus S_{m}$ with $e_{i} e_{j}=e_{i} \delta_{i, j}, S e_{i}$ connected for all $i, j$. Let $\sigma \in \operatorname{Aut}_{R}(S)$ and assume $\sigma$ induces the identity automorphism on $S / I S$. Then $\sigma\left(e_{i}\right)=e_{i}$ for all $i$ since $e_{i}+I S \neq e_{j}+I S$ for any $i \neq j$. Therefore $\sigma$ induces $\left(\sigma_{1}, \ldots, \sigma_{m}\right) \in \times_{i} \operatorname{Aut}_{R}\left(S_{i}\right)$. Since $\sigma$ is the identity on each of these summands, by the previous paragraph $\sigma$ is the identity on $S$ and $\rho$ is a one-to-one map.

(b) We can write $R=R_{1} \oplus \cdots \oplus R_{k}$, where each $R_{i}$ has a connected spectrum. Then there are the corresponding decompositions $S=S_{1} \oplus \cdots \oplus S_{k}$ and $I=I_{1} \oplus \cdots \oplus I_{k}$ with $I_{j}$ a nilpotent ideal of $R_{j}$ (in particular, no $I_{j}=R_{j}$ and $\operatorname{Aut}_{R}(S)=\times_{j} \operatorname{Aut}_{R_{j}}\left(S_{j}\right)$, $\left.\operatorname{Aut}_{R / I}(S / I S)=\times_{j} \operatorname{Aut}_{R / I_{j}}\left(R_{j} \otimes S / I_{j}\left(R_{j} \otimes S\right)\right)\right)$. Thus we can assume $R$ is connected.

If $I$ is nilpotent, then $I S$ is nilpotent and idempotents can be lifted modulo a nilpotent ideal, so $R / I$ has a connected spectrum and if $S$ is connected then $S / I S$ is connected. Assume $S$ is connected and Galois. Then $\operatorname{Aut}_{R}(S)=$ the Galois group of $S$ over $R=$ the Galois group $S / I S$ over $R / I=\operatorname{Aut}_{R / I}(S / I S)$ so $\rho$ is bijective in this case. If $S / R$ is Galois, then $S=S e_{1} \oplus \cdots \oplus S e_{m}$ with $e_{i} e_{j}=e_{i} \delta_{i, j}, S e_{i}$ connected, $S e_{i} \cong S e_{j}$ for all $i, j$, and each $S e_{i}$ Galois over $R$ with Galois group of order $n=\operatorname{rank}\left(S e_{i}\right)$. Thus $\left|\operatorname{Aut}_{R}(S)\right|=m ! n^{m}$. Since idempotents can be lifted modulo $I$, we get the same count for $\left|\operatorname{Aut}_{R / I}(S / I S)\right|$, so by part (a), $\rho$ is onto in this case.

If $S / R$ is finite étale, there is a Galois extension $N$ of $R$ containing $S$ constructed in the following way. Write $S=S e_{1} \oplus \cdots \oplus S e_{m}$ as above and let $L$ be a connected Galois extension of $R$ containing all the $S e_{i}$. Let $N=L e_{1} \oplus \cdots \oplus L e_{m}$. Let $\bar{\tau} \in \operatorname{Aut}_{R / I}(S / I S)$. Then one can extend $\bar{\tau}$ to $\bar{\gamma} \in \operatorname{Aut}_{R / I}(N / I N)$ which corresponds, by the paragraph above, to $\gamma \in \operatorname{Aut}_{R}(N)$. Then $(\gamma(S)+I S) / I=S / I$ so $\gamma(S) \subset S$. Therefore $\left.\gamma\right|_{S} \in \operatorname{Aut}_{R}(S)$ and $\rho\left(\left.\gamma\right|_{S}\right)=\bar{\tau}$. Thus $\rho$ is a bijection in every case.

COROLLARY 2.6. Let $X=\operatorname{Spec}(R)$, I the nil radical of $R$ and $X_{\mathrm{red}}=\operatorname{Spec}(R / I)$. If $S$ is a finite étale $R$-algebra, then $H^{1}(X, \operatorname{Aut}(\mathscr{S}))$ and $H^{1}\left(X_{\mathrm{red}}\right.$, Aut $\left.(\mathscr{S} / \mathscr{\Phi})\right)$ are bijective with one another.

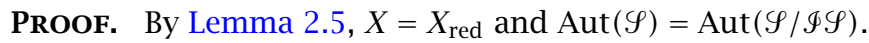

EXAMPLE 2.7. (a) Let $\mathbb{R}$ denote the real numbers and $\mathbb{C}$ the complex numbers. Let $R=\mathbb{R} \oplus \mathbb{R}$ and $S=\mathbb{C} \oplus \mathbb{C}$. Let $\sigma$ be complex conjugation. Then $(1,1)=(1, \sigma)$ on the first summand but $(1,1) \neq(1, \sigma)$ so the map $\operatorname{Aut}_{R}(S) \rightarrow \operatorname{Aut}_{R / I}(S / I S)$ is not always a one-to-one map.

(b) Let $R$ be the localization of $\mathbb{C}[x]$ at $(x)$ and let $p(t)=t^{3}+(x+1) \in R[t]$. Let 
$S=R[t] /(p(t))$. Since $p(t)$ is irreducible, $\operatorname{Aut}_{R}(S)=C_{3}$ (the cyclic group of order 3) but $R /(x)=\mathbb{C}$ and $S /(x) S=\mathbb{C} \oplus \mathbb{C} \oplus \mathbb{C}$ has $\mathbb{C}$-automorphism group $S_{3}$ (the symmetric group on three letters). It is not always the case that $\operatorname{Aut}_{R}(S) \rightarrow \operatorname{Aut}_{R / I}(S / I S)$ is onto.

MAYER-VIETORIS I. Let $R$ be a one-dimensional integral domain with module finite integral closure $\bar{R}$ and conductor $c=\{x \in R \mid \bar{R} \cdot x \subset R\}$. Let $S$ be a finite étale $R$ algebra. Assume the following.

(a) If $P$ is a maximal ideal in $R$ containing $c$ then there is only one maximal ideal $Q$ in $\bar{R}$ lying over $P$.

(b) If $P$ is a maximal ideal in $R$ containing $c$ then the natural map $\operatorname{Aut}_{R}(S) \rightarrow$ Aut $_{R / P}(S / P S)$ is surjective.

(c) $\bar{R} \otimes S \cong \bar{R}^{(n)}$, where $n=\operatorname{rank}_{R}(S)$.

Then there is an exact sequence of pointed sets

$$
\begin{gathered}
1 \longrightarrow \operatorname{Aut}_{R}(S) \stackrel{\alpha}{\longrightarrow} \operatorname{Aut}_{\bar{R}}(\bar{R} \otimes S) \times \operatorname{Aut}_{R / c}(R / c \otimes S) \\
\stackrel{\beta}{\longrightarrow} \operatorname{Aut}_{\bar{R} / c}(\bar{R} / c \otimes S) \stackrel{\gamma}{\longrightarrow} H^{1}(X, \operatorname{Aut}(\mathscr{S})) \longrightarrow 1 .
\end{gathered}
$$

SKetch OF THE PRoof. We define explicitly the maps in the sequence. Checking exactness at each term is then a straightforward computation.

The map $\alpha$ is given as $\alpha(\sigma)=(1 \otimes \sigma, 1 \otimes \sigma)$. The map $\beta$ is given as $\beta(\tau, \rho)=$ $(1 \otimes T)(1 \otimes \rho)^{-1}$.

Let $P_{1}, \ldots, P_{k}$ be the maximal ideals of $R$ lying over $c$. Using hypothesis (a), let $Q_{1}, \ldots, Q_{k}$ be the maximal ideals in $\bar{R}$ lying over $c$ with $Q_{i} \cap R=P_{i}(1 \leq i \leq k)$. Then $c=\cap_{i} P_{i}^{f_{i}}=\cap Q_{i}^{e_{i}}$ so $R / c=\oplus R / P^{f_{i}}$ and $\bar{R} / c=\oplus \bar{R} / Q^{e_{i}}$. Moreover, $\operatorname{Aut}_{R / c}(S / c S)=$ $\times_{i} \operatorname{Aut}_{R / P^{f_{i}}}\left(R / P_{i}^{f_{i}} \otimes S\right)$ and $\operatorname{Aut}_{\bar{R} / c}(\bar{R} / c \otimes S)=\times_{i} \operatorname{Aut}_{\bar{R} / Q^{e_{i}}}\left(\bar{R} / Q^{e_{i}} \otimes S\right)$. If $\left(\ldots, \bar{\sigma}_{i}, \ldots\right) \in$ $\operatorname{Aut}_{\bar{R} / c}(\bar{R} / c \otimes S)$ then by hypothesis (c) and Lemma 2.5, $\operatorname{Aut}_{\bar{R}}(\bar{R} \otimes S)=S_{n}=$

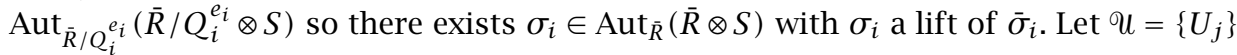
be a cover of $X=\operatorname{Spec}(R)$ with $P_{i} \in U_{j}$ if and only if $i=j$. Assign to $U_{j}$ the identity automorphism if no $P_{i}$ is in $U_{j}$. Since $U_{i} \cap U_{j}$ does not contain any points lying over $c$, Aut $\mathcal{O}_{\left(U_{i} \cap U_{j}\right)}\left(\mathscr{Y}\left(U_{i} \cap U_{j}\right)\right)=S_{n}$ and therefore contains the element $a(i, j)=\sigma_{i}^{-1} \sigma_{j}$. It is now easy to check $a \in Z^{1}(u$, Aut $(\mathscr{S}))$ is a 1-cocycle and a different choice of cover gives an equivalent cocycle modulo coboundaries, so $\gamma$ is defined by $\gamma\left(\ldots, \bar{\sigma}_{i}, \ldots\right)=$ $|a| \in H^{1}(X, \operatorname{Aut}(\mathscr{Y}))$.

MAYER-VIETORIS II. Let $R$ be a reduced ring with $X=\operatorname{Spec}(R)$ connected. Let $I_{1}, \ldots, I_{q}$ be the set of minimal prime ideals of $R$ and $\bar{R}=\oplus_{j=1}^{q} R / I_{j}$. Assume $\operatorname{dim} R / I_{j}=1$ for all $j$. Identify $R$ with its natural image in $\bar{R}$ and let $c=\{x \in R \mid \bar{R} \cdot x \subset R\}$ be the conductor. Let $Y=\operatorname{Spec}(\bar{R})$. Let $S$ be a finite étale $R$-algebra.

(a) Assume for each maximal ideal $Q$ in $\bar{R}$ lying over $c$ the natural map $\operatorname{Aut}_{R}(S) \rightarrow$ $\operatorname{Aut}_{\bar{R} / Q}(\bar{R} / Q \otimes S)$ is a surjection.

Then there is an exact sequence of pointed sets

$$
\begin{aligned}
& 1 \longrightarrow \operatorname{Aut}_{R}(S) \stackrel{\alpha}{\longrightarrow} \operatorname{Aut}_{\bar{R}}(\bar{R} \otimes S) \times \operatorname{Aut}_{R / c}(R / c \otimes S) \\
& \stackrel{\beta}{\longrightarrow} \operatorname{Aut}_{\bar{R} / c}(\bar{R} / c \otimes S) \stackrel{\gamma}{\longrightarrow} H^{1}(X, \operatorname{Aut}(\mathscr{Y})) \stackrel{\delta}{\longrightarrow} H^{1}(Y, \operatorname{Aut}(\bar{R} \otimes \mathscr{S})) .
\end{aligned}
$$


SKeTCH OF THE PROOF. As in Mayer-Vietoris I, we give the maps explicitly, then checking exactness is a straightforward computation. The map $\alpha$ is defined as $\alpha(\sigma)=$ $(1 \otimes \sigma, 1 \otimes \sigma)$. The map $\beta$ is $\beta(\tau, \rho)=(1 \otimes \tau)(1 \otimes \rho)^{-1}$.

Let $P_{1}, \ldots, P_{m}$ be the maximal ideals in $R$ lying over $c$ and $Q_{i, j}$ the maximal ideals in $\bar{R}$ lying over $c$ where the projection of $Q_{i, j}$ on $R / I_{j}$ is proper. Write $c=\cap_{k=1}^{m} P_{k}^{f_{k}}=\cap_{i, j} Q_{i, j}^{e_{i, j}}$. Then $\operatorname{Aut}_{\bar{R}}(\bar{R} \otimes S)=\times_{j=1}^{q} \operatorname{Aut}_{R / I_{j}}\left(R / I_{j} \otimes S\right), \operatorname{Aut}_{R / c}(R / c \otimes S)=\times_{k=1}^{m} \operatorname{Aut}_{R / P_{k}^{f_{k}}}\left(R / P_{k}^{f_{k}} \otimes S\right)$, and $\operatorname{Aut}_{\bar{R} / c}(\bar{R} / c \otimes S)=\times_{i, j} \operatorname{Aut}_{\bar{R} / Q_{i, j}} e_{i, j}\left(\bar{R} / Q_{i, j}^{e_{i, j}} \otimes S\right)$. Let $\left(\ldots, \bar{\sigma}_{i, j}, \ldots\right) \in \operatorname{Aut}_{\bar{R} / c}(\bar{R} / c \otimes S)=$ $\times_{i, j} \operatorname{Aut}_{\bar{R} / Q_{i, j}^{e_{i, j}}}\left(\bar{R} / Q_{i, j}^{e_{i, j}} \otimes S\right)$. By hypothesis (a) there is $\sigma_{i, j} \in \operatorname{Aut}_{R / I_{i}}\left(R / I_{i} \otimes S\right)$ which reduces to $\bar{\sigma}_{i, j}$. For a fixed $i,\left\{\sigma_{i, j}\right\}$ determines an element $\sigma_{i} \in \operatorname{Aut}_{\bar{R}}(\bar{R} \otimes S)$ where we let $\sigma_{i}$ be the identity in $\operatorname{Aut}_{R / I_{k}}\left(R / I_{k} \otimes S\right)$ if $k$ is not any $j$. Let $U=\left\{U_{i}\right\}$ be an open cover of $X=\operatorname{Spec}(R)$ where $P_{i} \in U_{j}$ if and only if $i=j$. Let $\gamma\left(\ldots, \bar{\sigma}_{i, j}, \ldots\right)=|a| \in H^{1}(X$, Aut $(\mathscr{S}))$ where $a \in Z^{1}(\mathcal{U}$, Aut $(\mathscr{Y}))$ is given by $a(i, k)=\sigma_{i}^{-1} \sigma_{k} \in \operatorname{Aut}_{\mathscr{O}\left(U_{i} \cap U_{k}\right)}\left(\mathcal{O}_{(}\left(U_{i} \cap U_{k}\right) \otimes S\right)$. Note, $\sigma_{i}^{-1} \sigma_{k}$ is defined since $U_{i} \cap U_{k}$ contains no $P_{j}$ so $\mathcal{O}\left(U_{i} \cap U_{j}\right) \otimes S=\mathcal{O}\left(U_{i} \cap U_{j}\right) \otimes_{\bar{R}} \bar{R} \otimes S$. It is clear that the definition of $\gamma$ is independent of the choice of cover and our assignment gives a well-defined map.

Let $U=\left\{U_{i}\right\}$ be an open cover of $X$ constructed as above and let $\pi: Y \rightarrow X$ be given by restriction. Let $V_{i}=\pi^{-1}\left(U_{i}\right)$ so $\mathscr{V}=\left\{V_{i}\right\}$ is an open cover of $Y$. Given $a \in$ $Z^{1}(\mathcal{U}$,Aut $(\mathscr{S}))$, let $\delta(a) \in Z^{1}(\mathcal{V}, \operatorname{Aut}(\bar{R} \otimes \mathscr{S}))$ by $\delta(a)(i, j)=a(i, j)$. This assignment is well defined since $\mathscr{O}_{X}\left(U_{i} \cap U_{j}\right)=\mathscr{O}_{Y}\left(V_{i} \cap V_{j}\right)$.

Note 2.8. If each $R / I_{j}$ in Mayer-Vietoris II is integrally closed, $H^{1}(Y,(\bar{R} \otimes \mathscr{S}))=\{1\}$ by Proposition 2.3. This is the case in all the following examples.

EXAMPLE 2.9. Let $\mathbb{Q}$ denote the rational numbers, let $p, q$ be prime integers, and let $\omega$ be a primitive complex $p$ th root of 1 . Let $F=\mathbb{Q}(\omega)$ and $R=F[x, y] /\left(x^{p}-q y^{p}(y-1)^{p}\right)$. If $q$ is irreducible in $\mathbb{Z}[\omega]$ then by Eisenstein's criterion $x^{p}-q y^{p}(y-1)^{p}$ is irreducible in $F[x, y]$ so $R$ is a one-dimensional Noetherian integral domain. Note that $q$ is irreducible in $\mathbb{Z}[\omega]$ whenever $p \nmid q-1, p \neq q$. Let $S=R[t] /\left(t^{p}-q\right)$. Then $S$ is a finite étale $R$-algebra which is connected since $t^{p}-q$ is irreducible in $F[t]$ and $R /(x, y)=F$. Identify $x, y$ with their images in $R$. The integral closure $\bar{R}$ of $R$ is $R(x / y(y-1))$ and since $(x / y(y-1))^{p}=q, t^{p}-q=\Pi_{i=0}^{p-1}\left(t-\omega^{i}(x /(y(y-1)))\right) \in \bar{R}[t]$ so $\bar{R} \otimes S \cong \bar{R}^{(p)}$. The maximal ideals lying over $c$ in $R$ are $(x, y)$ and $(x, y-1)$ and the only maximal ideal in $\bar{R}$ lying over $(x, y)$ is $(y)$, the only maximal ideal in $\bar{R}$ lying over $(x, y-1)$ is $(y-1)$. Since $R /(x, y) \cong F$, and $R /(x, y-1) \cong F$, and $t^{p}-q$ is irreducible in $F[t], R$ satisfies the hypothesis of Mayer-Vietoris I. $\operatorname{But}_{\operatorname{Aut}_{R}}(S)=C_{p}$, the cyclic group of order $p$. $\operatorname{Aut}_{\bar{R}}(\bar{R} \otimes S)=S_{p}$, the symmetric group on $p$-letters. Aut $\operatorname{Au}_{R / c}(R / c \otimes S)=C_{p} \times C_{p}$ and $\operatorname{Aut}_{\bar{R} / c}(\bar{R} / c \otimes S)=S_{p} \times S_{p}$ so for this example the Mayer-Vietoris I sequence becomes

$$
1 \rightarrow C_{p} \rightarrow S_{p} \times\left(C_{p} \times C_{P}\right) \rightarrow S_{p} \times S_{p} \rightarrow H^{1}(X, \operatorname{Aut}(\mathscr{S})) \rightarrow 1
$$

Let $K=\left\{\left(\tau \rho^{-1}, \tau \sigma^{-1}\right) \mid \tau \in S_{p}, \rho, \sigma \in C_{p}\right\}$. Then $H^{1}(X, \operatorname{Aut}(\mathscr{S}))$ is bijective with the coset space $S_{p} \times S_{p} / K$ and has order $(p-1)$ !. In particular, when $p=2$ there are no nontrivial twisted forms, when $p=3$ there is exactly one nontrivial twisted form, and so forth. 
EXAMPLE 2.10. (a) Let $R$ be a Noetherian domain with quotient field $K$, assume $R$ contains a primitive $n$th root of 1 and $I$ is a fractional $R$-ideal in $K$ with $I^{n}=R$. Assume $p(t)=t^{n}-a \in R[t]$ is a separable polynomial, and $S=R[t] /(p(t)), L=K[t] /(p(t))$. Then the subset $T=\oplus_{j=0}^{n-1} I^{j} t^{j}$ of $L$ is a twisted form of $S$.

(b) Let $R$ be a reduced Noetherian ring with minimal prime ideals $I_{1}, \ldots, I_{q}$ and assume the dimension of each $R / I_{j}$ is one. Let $K=\oplus R / I_{j}$. Assume $R$ contains a primitive $n$th root of 1 and $I$ is a finitely generated $R$-submodule of $K$ with $I^{n}=R$. Assume $p(t)=t^{n}-a \in R[t]$ is a separable polynomial and $S=R[t] /(p(t)), L=K[t] /(p(t))$. Then the subset $T=\oplus_{j=0}^{n-1} I^{j} t^{j}$ of $L$ is a twisted form of $S$.

Note 2.11. In Example 2.9 if $p=3$ and $q=2$ then the nontrivial twisted form $T$ is constructed as in Example 2.10 where the ideal $I=\left(y, y^{-1} x\right)$, as one can check by showing the associated cocycle in $H^{1}(X$, Aut $(\mathscr{Y}))$ is not a coboundary. Notice $T$ is free as an $R$-module. Let $\mathscr{F}_{(}(S)$ be the set of isomorphism classes of twisted forms of $S$ which are free as $R$-modules and assume $S$ is free as an $R$-module. Then there is an exact sequence of pointed sets $1 \rightarrow \mathscr{F}(S) \rightarrow H^{1}(X, \operatorname{Aut}(\mathscr{S})) \rightarrow H^{1}(X, \mathrm{Gl}(\mathscr{Y}))$. The types of examples given in Example 2.10 all lie in $\mathscr{F}(S)$, but we give in Example 2.12 a twisted form of $S$ whose image in $H^{1}(X, \mathrm{Gl}(\mathscr{S}))$ is not the identity.

EXAMPLE 2.12. Let $R=\mathbb{R}[x, y] /(y-1)\left(y-x^{2}\right)$ and $\bar{R}=\mathbb{R}[x, y] /(y-1) \oplus$ $\mathbb{R}[x, y] /\left(y-x^{2}\right)$, where $\mathbb{R}$ is the set of the real numbers. Then $R=\{(p(x, y), q(x, y)) \in$ $\bar{R} \mid p(1,1)=q(1,1) ; p(-1,1)=q(-1,1)\}$. Let $I=\{(p(x, y), q(x, y)) \in \bar{R} \mid p(1,1)=$ $q(1,1) ; p(-1,1)=-q(-1,1)\}$. Then $I$ is an $R$-submodule of $\bar{R}, I^{2}=R$, and $R_{P} \otimes I \cong R_{P}$ for all prime ideals $P$ of $R$. Let $p(t)=t^{2}+1$, let $S=R[t] /\left(t^{2}+1\right)$, and take $T=R \oplus I t$. Then $T$ is a twisted form of $S$ and $T$ is not a free $R$-module by cancellation [9] so $T$ is a nontrivial twisted form of $S$. Notice that $T$ is a Galois extension of $R$ with Galois group of order two induced by complex conjugation but since $T$ is not free, $T$ does not have either a normal basis or a primitive element.

EXAMPLE 2.13. Consider $T$ as constructed in Example 2.12 and let $w^{2}+1 \in T[w]$. Then $q(w)=w^{2}+1$ is irreducible in $T[w]$ but for each prime ideal $Q$ of $T, q(w)$ is reducible in $T_{Q}[w]$. This gives an example of an irreducible separable polynomial over a connected commutative ring which factors into linear factors over the localization at every prime ideal or modulo each maximal ideal. If $r(w)=w^{2}-1$ then $S_{1}=R[w] /(q(w))$ is not isomorphic to $S_{2}=R[w] /(r(w))$ since $S_{2} \cong R \oplus R$ but $S_{1}$ and $S_{2}$ are locally isomorphic. This is an example of two separable polynomials that are locally isomorphic but not isomorphic (in the sense of [4]).

EXAMPLE 2.14. Let $R=\mathbb{Q}[x, y] /(y-1)\left(y-x^{2}\right)$ as in Example 2.12. Let $p(t)=$ $t^{3}-3 t+1$ and $S=R[t] /(p(t))$. Using the Mayer-Vietoris sequence for the Picard group, [1] or [5], one can check the torsion part of the Picard group is $C_{2}$. But MayerVietoris II gives $H^{1}(X$, Aut $(\mathscr{Y}))=C_{3}$ so if $T$ is a nontrivial twisted form of $S$, then $T$ is not isomorphic to $R \oplus I t \oplus I t^{2}$ for any fractional ideal of $R$ with $I^{3}=R$.

EXAMPLE 2.15. If $R$ is as in Example 2.14 and $p(t)=t^{3}-2$, then one can check that $p(t)$ is separable and $p(t)$ factors into linear factors modulo each minimal prime 
ideal of $R$ but $p(t)$ is irreducible in $R[t]$. Hypothesis (a) of Mayer-Vietoris II fails to hold for this example.

ACKNOWLEDGEMENT. This article was written while the author was a visitor at the Eidgenossische Technische Hochschule (ETH) in Zurich, Switzerland. I would like to thank Max Knus and Beno Eckmann of the ETH for several stimulating conversations.

\section{REFERENCES}

[1] H. Bass, Algebraic K-Theory, Mathematics Lecture Note Series, vol. 15, W. A. Benjamin, New York, 1968. MR 40\#2736. Zbl 174.30302.

[2] F. DeMeyer and E. Ingraham, Separable Algebras over Commutative Rings, Lecture Notes in Mathematics, vol. 181, Springer-Verlag, New York, 1971. MR 43\#6199. Zbl 215.36602.

[3] J. Giraud, Cohomologie Non Abélienne, Die Grundlehren der Mathematischen Wissenschaften, vol. 179, Springer-Verlag, Berlin, 1971 (French). MR 49\#8992. Zbl 226.14011.

[4] D. K. Harrison and T. McKenzie, Toward an arithmetic of polynomials, Aequationes Math. 43 (1992), no. 1, 21-37. MR 92m:13008. Zbl 759.13005.

[5] R. Hartshorne, Algebraic Geometry, Graduate Texts in Mathematics, no. 52, SpringerVerlag, New York, 1977. MR 57\#3116. Zbl 367.14001.

[6] G. J. Janusz, Separable algebras over commutative rings, Trans. Amer. Math. Soc. 122 (1966), 461-479. MR 35\#1585. Zbl 141.03402.

[7] M.-A. Knus, Quadratic and Hermitian Forms over Rings, Grundlehren der Mathematischen Wissenschaften, vol. 294, Springer-Verlag, Berlin, 1991. MR 92i:11039. Zbl 756.11008.

[8] J. S. Milne, Étale Cohomology, Princeton Mathematical Series, vol. 33, Princeton University Press, New Jersey, 1980. MR 81j:14002. Zbl 433.14012.

[9] J.-P. Serre, Modules projectifs et espaces fibrés à fibre vectorielle, Séminaire P. Dubreil, M.-L. Dubreil-Jacotin, et C. Pisot, 1957/58, Algebre Theorie Nombres, no. 23, Secrétariat Mathématique, Paris, 1958 (French). MR 31\#1277. Zbl 132.41202.

Frank DeMeyer: Department of Mathematics, Colorado State University, Fort COLLINS, CO 80523, USA

E-mail address: demeyer@math.colostate.edu 


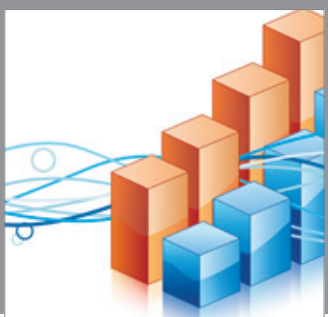

Advances in

Operations Research

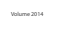

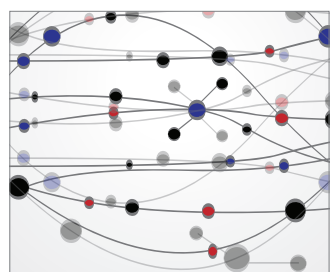

\section{The Scientific} World Journal
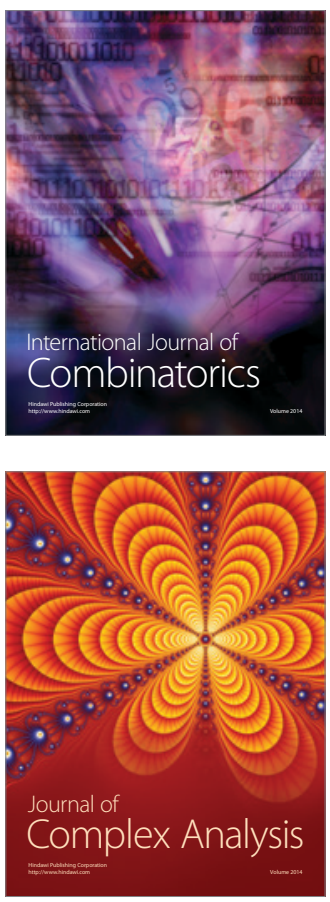

International Journal of

Mathematics and

Mathematical

Sciences
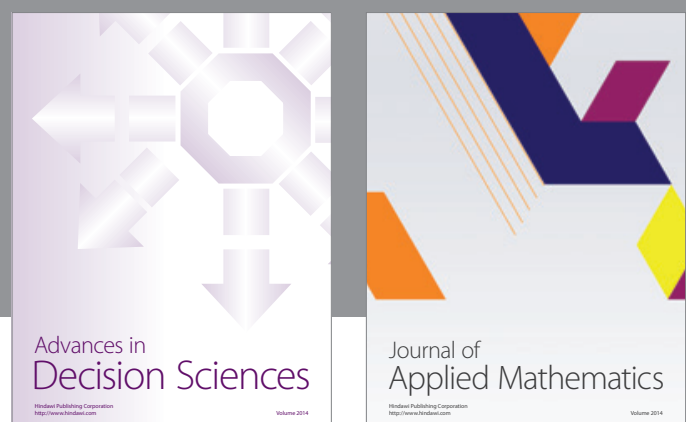

Journal of

Applied Mathematics
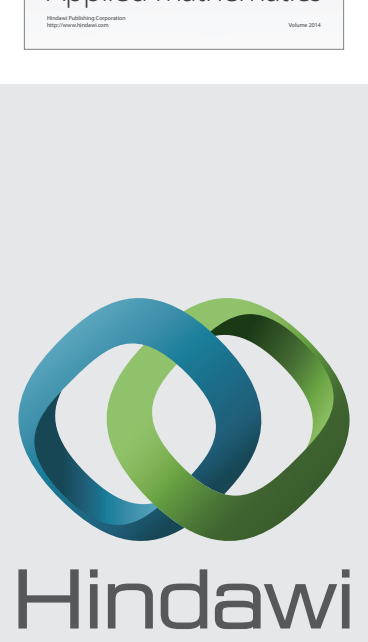

Submit your manuscripts at http://www.hindawi.com
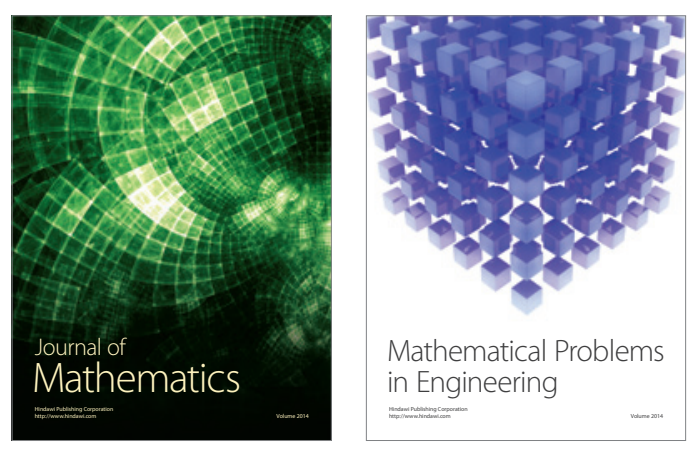

Mathematical Problems in Engineering
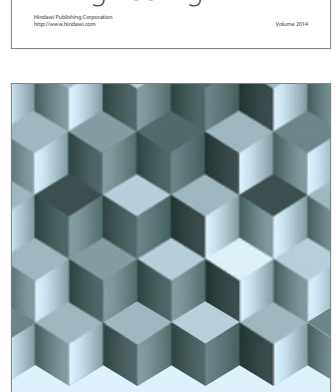

Journal of

Function Spaces
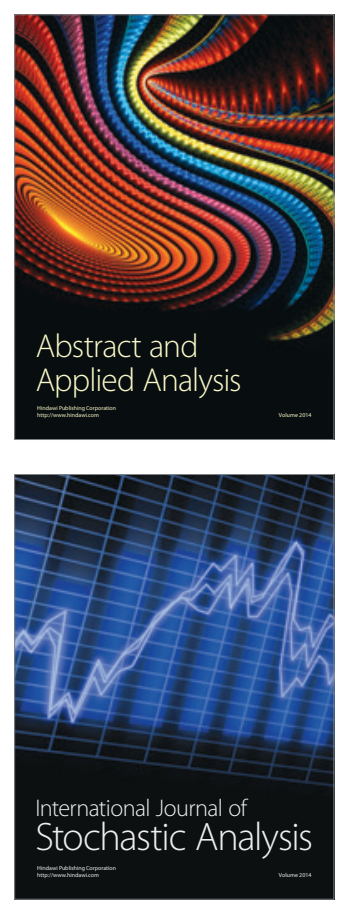

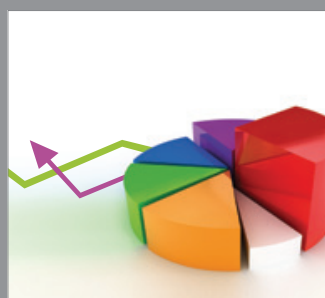

ournal of

Probability and Statistics

Promensencen
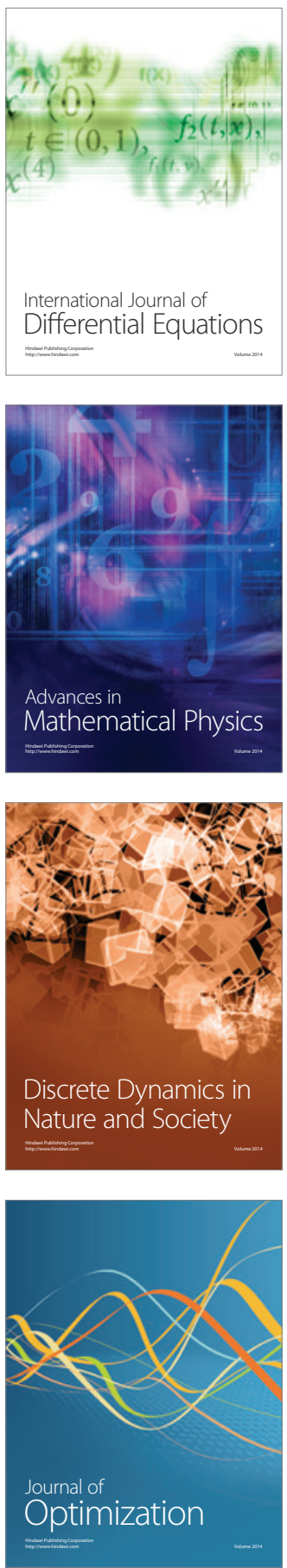